\title{
Intensive Care Unit Relocation and Its Effect on Multidrug-Resistant Respiratory Microorganisms
}

\author{
Hyung-Jun Kim', EuiSeok Jeong ${ }^{2}$, Pyoeng Gyun Choe' ${ }^{2}$ Sang-Min Lee', Jinwoo Lee' \\ 'Division of Pulmonary and Critical Care Medicine, Department of Internal Medicine and I'Infection Control Center, Seoul National University Hospital, Seoul, \\ Korea
}

Background: Infection by multidrug-resistant (MDR) pathogens leads to poor patient outcomes in intensive care units (ICUs). Contact precautions are necessary to reduce the transmission of MDR pathogens. However, the importance of the surrounding environment is not well known. We studied the effects of ICU relocation on MDR respiratory pathogen detection rates and patient outcomes.

Methods: Patients admitted to the ICU before and after the relocation were retrospectively analyzed. Baseline patient characteristics, types of respiratory pathogens detected, antibiotics used, and patient outcomes were measured.

Results: A total of 463 adult patients admitted to the ICU, 4 months before and after the relocation, were included. Of them, 234 were admitted to the ICU before the relocation and 229 afterward. Baseline characteristics, including age, sex, and underlying comorbidities, did not differ between the two groups. After the relocation, the incidence rate of MDR respiratory pathogen detection decreased from 90.0 to 68.8 cases per 1,000 patient-days, but that difference was statistically insignificant. The use of colistin was significantly reduced from 53.5 days (95\% confidence interval [Cl], 20.3 to 86.7 days) to 18.7 days (95\% Cl, 5.6 to 31.7 days). Furthermore, the duration of hospital stay was significantly reduced from a median of 29 days (interquartile range [IQR], 14 to 50 days) to 21 days (IQR, 11 to 39 days).

Conclusions: Incidence rates of MDR respiratory pathogen detection were not significantly different before and after ICU relocation. However, ICU relocation could be helpful in reducing the use of antibiotics against MDR pathogens and improving patient outcomes.

Key Words: environment; intensive care units; multiple drug resistance

\section{INTRODUCTION}

Given the extensive use of antibiotics in intensive care units (ICUs) [1], nosocomial infections with multidrug-resistant (MDR) pathogens in an ICU have become a major problem. In a previous prospective surveillance study of 10 Asian countries, resistance to imipenem was observed in $67.3 \%$ of Acinetobacter isolates and $27.2 \%$ of Pseudomonas aeruginosa isolates, with MDR rates of $82 \%$ and $42.8 \%$ among patients with hospital-acquired pneumonia and ventilator-associated pneumonia, respectively [2]. The mortality rates of hospital-acquired pneumonia and ventilator-associated pneumonia are higher than those of community-acquired pneumonia and healthcare-associated pneumonia [3]. Furthermore, MDR pathogens

\section{Original Article}

Received: June 18, 2018

Revised: August 20, 2018

Accepted: September 10, 2018

\section{Corresponding author}

Jinwoo Lee

Division of Pulmonary and Critical Care Medicine, Department of Internal Medicine, Seoul National University Hospital, 101 Daehak-ro, Jongno-gu, Seoul 03080, Korea Tel: +82-2-2072-7593

Fax: +82-2-762-9662

E-mail: realrain7@gmail.com

Copyright () 2018 The Korean Society of Critical Care Medicine

This is an Open Access article distributed under the terms of Creative Attributions Non-Commercial License (http:// creativecommons.org/li-censes/by-nc/4.0/) which permits unrestricted noncommercial use, distribution, and reproduction in any medium, provided the original work is properly cited. 
can increase the mortality rates of ICU-acquired pneumonia [4]. Therefore, controlling the prevalence of MDR pathogens in the ICU is mandatory for patient safety.

Efforts have been made to decrease the risk of MDR pathogen transmission. Methods such as active surveillance cultures, room placement, cohorting, hand hygiene, use of clean gloves and gowns, and enforcing strict adherence to precautions have been used to decrease the incidence rates (IRs) of MDR pathogen infections [5-7]. However, all those precautions focus on person-to-person transmission. The importance of environmental contamination is not well understood. In the present study, we elucidate the effects of an environmental change, the relocation of a medical ICU (MICU), on respiratory MDR microorganisms in the ICU.

\section{MATERIALS AND METHODS}

\section{Study Population and Clinical Characteristics}

Between November 2016 and July 2017, patients admitted to the MICU in a tertiary referral center in Seoul National University Hospital were screened. All adult patients ( $\geq 18$ years) were included. Because of a remodel of the center's MICU, patients had to be moved to a newly-built temporary MICU on the 8th of March, 2017. Prior to its relocation, the MICU had 20 open beds and two isolation beds, and the average space was $19.7 \mathrm{~m}^{2}$ per patient. Among the 20 open beds, four belonged to the Department of Neurology and were excluded from the analysis. After its relocation, the MICU had 12 open beds with two isolation beds, all of which belonged to the Department of Internal Medicine, and the average space was $21.7 \mathrm{~m}^{2}$ per patient.

Patients were retrospectively analyzed in terms of baseline demographics (sex, age, underlying comorbidities, and Acute Physiology and Chronic Health Evaluation [APACHE] II scores) before and after the relocation. The use of medications with potential effects on respiratory microbiota, such as antacids and steroids, and antibiotic use were also reviewed. As clinical outcomes, we compared the use of antibiotics, length of MICU stay, length of hospital stay, and in-hospital mortality. Days of medication use and ventilator use were calculated in calendar days.

This study was conducted in accordance with the amended Declaration of Helsinki and was approved by the Institutional Review Board of Seoul National University Hospital (protocol no. H-1705-019-851). The requirement for informed consent was waived because of the retrospective study design and because all patients records and information were anonymized prior to analysis.

\section{KEY MESSAGES}

- Multi-drug resistant respiratory pathogens tended to decrease after relocation of the intensive care unit, although without statistical significance.

- Use of colistin was significantly reduced after the relocation.

- Duration of hospital stay was shorter after the relocation.

\section{Microbiological Evaluation}

To maximize the sensitivity of respiratory microbiome detection, several respiratory specimen culture results were reviewed. Noninvasive respiratory specimens from sputum and endotracheal aspirate, along with invasive respiratory specimens such as bronchial washings and bronchoalveolar lavage fluids were included. Sputum was collected in a sterile conical tube after coughing effort from the patient, and endotracheal aspiration was performed by the attending nurse with a sterile catheter and saline. Bronchoscopic specimens were collected by a board-certified pulmonary specialist with a sterile bronchoscope and saline. Active surveillance cultures of carbapenem-resistant Acinetobacter baumannii (CRAB) were routinely performed from sputum or endotracheal aspirate specimens upon MICU admission and were also included in the analysis. CRAB, methicillin-resistant Staphylococcus aureus (MRSA), carbapenem-resistant Pseudomonas aeruginosa (CRPA), extended-spectrum $\beta$-lactamase (ESBL)-producing Gram-negative Enterobacteriaceae, and Stenotrophomonas maltophilia were considered as the MDR pathogens [8,9]. The IR of MDR respiratory pathogens was compared before and after the relocation and also described monthly. The microorganisms were considered to be MICU-acquired if they were first detected after MICU admission, or if they first appeared after 3 days of MICU stay.

\section{Standard Precautions in the MICU}

Isolation beds were isolated with walls and a door, whereas open beds were divided by curtains. The patient to nurse ratio was 2:1 during the day and evening and 3:1 at night. Routine cleaning of the floors was performed every day with $4 \%$ sodium hypochlorite and was done more frequently when visual contamination was observed. When a patient left the ICU bed, every surface of the patient's medical equipment was cleaned with $50 \%$ benzalkonium chloride.

Medical professionals were required to wash their hands with soap and water and with $0.5 \%$ chlorhexidine gluconate in $70 \%$ or $83 \%$ ethanol before and after attending a patient. If any MDR microorganism was detected in a patient, wearing 
clean nonsterile gloves and gowns was obligatory while attending to that patient. Also, separate items such as thermometers, stethoscopes, tourniquets, and blood pressure cuffs were used. Adherence to those precautions was monitored on a weekly basis, and the precaution protocols did not change before and after the MICU relocation.

\section{Statistical Analysis}

Categorical variables are described as numbers and percentages and compared using either the chi-square test or Fisher exact test. Numerical variables are described using the median and interquartile range (IQR) and compared using the MannWhitney $U$-test. IRs are described as numbers per 1,000 patient-days with $95 \%$ confidence intervals (CIs) and compared using Poisson regression analyses or negative binomial regression analyses. All statistical analyses were performed using STATA version 13.0 (Stata Corp., College Station, TX, USA). The P-values of less than 0.05 were considered statistically significant.

\section{RESULTS}

\section{Baseline Characteristics}

A total of 463 patients were admitted to the MICU during the study period. Among them, 234 patients were admitted to the MICU before its relocation, and the other 229 were admitted afterward. Patients were predominantly male (65\%), with a median age of 66 years (IQR, 58 to 74 years) and a median APACHE II score of 28 (IQR, 19 to 36). Among the 463 patients, 108 (23.3\%) were diagnosed with sepsis, and 155 (33.5\%) were diagnosed with pneumonia. The prevalence of sepsis increased significantly after the MICU relocation ( $18.8 \%$ to $28.0 \%, \mathrm{P}=0.020$ ). The most common underlying comorbidity was hematologic malignancy (21.8\%), followed by solid organ malignancy (19.7\%), diabetes mellitus (13.4\%), heart failure (10.8\%), end-stage renal disease (9.7\%), liver cirrhosis (6.1\%), solid organ transplant (5.6\%), and bronchiectasis (3.5\%). The proportion of patients who were transferred from other ICUs was $18.8 \%$, which did not differ before and after the relocation $(\mathrm{P}=0.333)$. Antacids

Table 1. Baseline characteristics of 463 patients admitted to the medical ICU before and after its relocation

\begin{tabular}{|c|c|c|c|}
\hline Variable & Before relocation $(n=234)$ & After relocation $(n=229)$ & P-value \\
\hline Male sex & $154(65.8)$ & $147(64.2)$ & 0.715 \\
\hline Age (yr) & $67(59-75)$ & $65(57-74)$ & 0.233 \\
\hline APACHE II score & $27(19-34)$ & $29(19-38)$ & 0.166 \\
\hline Sepsis & 44 (18.8) & $64(28.0)$ & 0.020 \\
\hline Pneumonia & $69(29.5)$ & $86(37.6)$ & 0.066 \\
\hline \multicolumn{4}{|l|}{ Underlying disease } \\
\hline Hematologic malignancy & 45 (19.2) & $56(24.5)$ & 0.174 \\
\hline Solid organ malignancy & $46(19.7)$ & 45 (19.7) & 0.998 \\
\hline Diabetes mellitus & $32(13.7)$ & $30(13.1)$ & 0.856 \\
\hline Heart failure & $23(9.8)$ & 27 (11.8) & 0.497 \\
\hline End stage renal disease & $21(9.0)$ & $24(10.5)$ & 0.584 \\
\hline Liver cirrhosis & $12(5.1)$ & $16(7.0)$ & 0.402 \\
\hline Solid organ transplant & $15(6.4)$ & $11(4.8)$ & 0.453 \\
\hline Bronchiectasis & $8(3.4)$ & $8(3.5)$ & 0.965 \\
\hline Transferred from other ICU & $48(20.5)$ & 39 (17.0) & 0.333 \\
\hline Mechanical ventilator applied & $168(71.8)$ & $146(63.8)$ & 0.064 \\
\hline Ventilator day ${ }^{a}$ & $5(3-9)$ & $4(2-8)$ & 0.120 \\
\hline Use of antacids & 207 (88.5) & 199 (86.9) & 0.609 \\
\hline Use of steroids & $133(56.8)$ & $118(51.5)$ & 0.252 \\
\hline Steroid day ${ }^{a}$ & $4(2-8)$ & $4(2-7)$ & 0.883 \\
\hline No. of respiratory samples ${ }^{b}$ & 530.5 (488.2-572.8) & $513.5(468.6-558.5)$ & 0.588 \\
\hline
\end{tabular}

Value are presented as number (\%) or median (interquartile range) unless otherwise indicated.

ICU: intensive care unit; APACHE: Acute Physiology and Chronic Health Evaluation.

${ }^{a}$ Calculated as calendar days; ${ }^{b}$ Presented as incidence rate per 1,000 patient-days (95\% confidence interval). 
were administered to $87.7 \%$ of patients. Mechanical ventilation was administered to $67.8 \%$ of patients for a median of 4 days (IQR, 2 to 9 days), and ventilator dependency (ventilator use $\geq 21$ days) was observed in 43 of 483 patients ( $8.9 \%$ ), which did not differ before and after the MICU relocation (5.1\% vs. $4.8 \%, \mathrm{P}=0.872$ ). Steroids were administered to $54.2 \%$ of patients for a median of 4 days (IQR, 2 to 8 days). These baseline characteristics did not differ between the before and after MICU relocation groups. A total of 530.5 respiratory samples (95\% CI, 488.2 to 572.8) were used to perform microbiological analyses per 1,000 patient-days before the relocation, and 513.5 samples ( $95 \%$ CI, 468.6 to 558.5) were used per 1,000 patientdays after the relocation, and that difference was not statistically significant $(\mathrm{P}=0.558)$ (Table 1$)$.

\section{Changes in the MDR Respiratory Microorganisms}

The IR of MDR respiratory microorganism infections decreased from 90.0 to 68.8 per 1,000 patient-days after the MICU relocation, but that difference was not statistically significant $(\mathrm{P}=0.349)$. The IR of CRAB decreased from 49.2 to 30.7 per 1,000 patientdays $(\mathrm{P}=0.252)$, and the IR of MICU-acquired CRAB decreased from 32.5 to 26.8 per 1,000 patient-days $(\mathrm{P}=0.673)$. The IR of MRSA decreased from 25.6 to 19.0 per 1,000 patient-days $(\mathrm{P}=$ 0.530 ), and the IR of MICU-acquired MRSA decreased from 18.2 to 10.1 per 1,000 patient-days $(\mathrm{P}=0.337)$. Changes in other MDR respiratory microorganisms, including S. maltophilia, ESBL-producing gram-negative Enterobacteriaceae, and CRPA, were also not statistically significant (Figure 1).

Patients with sepsis and pneumonia were analyzed sepa- rately, but the results were similar. In patients with sepsis, the IR of all MDR pathogens decreased from 103.6 to 82.8 per 1,000 patient-days $(\mathrm{P}=0.672)$, and the IR of CRAB decreased from 87.8 to 35.5 per 1,000 patient-days $(\mathrm{P}=0.164)$, both lacking statistical significances. Among patients diagnosed with pneumonia, the IRs were similar: all MDR pathogens stayed the same (86.0 to 86.0 per 1,000 patient-days, $\mathrm{P}=0.992$ ), and CRAB decreased without significance (51.9 to 48.2 per 1,000 patient-days, $\mathrm{P}=0.909$ ).

The monthly IRs of MDR respiratory microorganisms were specified to further assess the effect of the MICU relocation. In contrast to a high IR of MDR respiratory microorganisms in February 2017 (125.9 per 1,000 patient-days), the IR in March 2017, the month in which the relocation of the MICU occurred, decreased to 70.5 per 1,000 patient-days. The IR further decreased to 33.8 per 1,000 patient-days in May 2017, and the difference between February 2017 and May 2017 was statistically significant $(\mathrm{P}=0.022)$ (Figure 2$)$. However, the IR rose again to 149.8 per 1,000 patient-days in June 2017, when 16 of 43 patients (37.2\%) were diagnosed with at least one detectable MDR pathogen. This detection rate was significantly higher than that observed for the other months, including April (3/44 patients, 6.8\%) and May (6/55 patients, 10.9\%). High rates of CRAB (7/43 patients, $16.3 \%$ ) and MRSA (5/43 patients, 11.6\%) detection were the main factors contributing to the increase in MDR microorganisms. Other than CRAB and MRSA, CRPA (two patients), and ESBL-producing gram-negative Enterobacteriaceae (two patients) were also detected in June 2017.

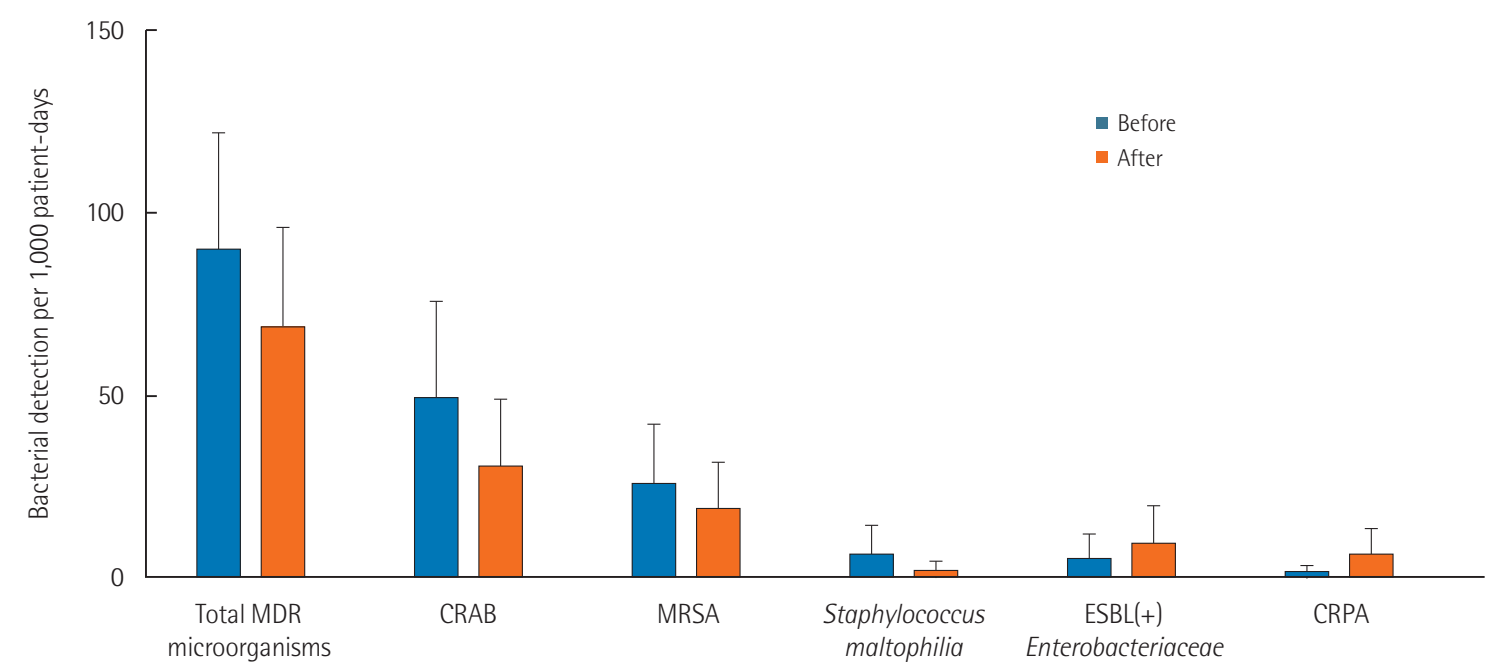

Figure 1. Changes in multidrug-resistant (MDR) microorganisms from the respiratory tract before and after the medical intensive care unit relocation. Bars indicate 95\% confidence intervals. CRAB: carbapenem-resistant Acinetobacter baumannii; MRSA: methicillin-resistant Staphylococcus aureus; ESBL: extended-spectrum $\beta$-lactamase; CRPA: carbapenem-resistant Pseudomonas aeruginosa. 


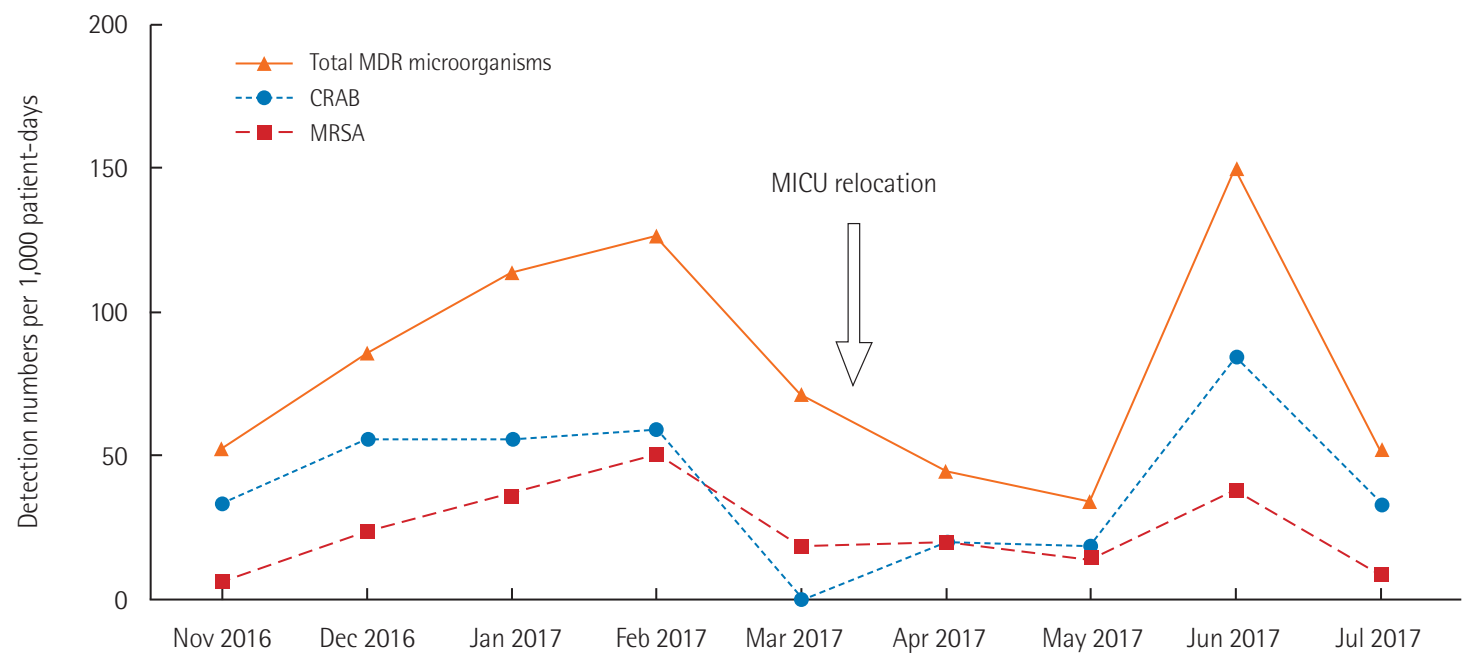

Figure 2. Monthly incidence rates of multidrug-resistant (MDR) microorganisms from the respiratory tract. CRAB: carbapenem-resistant Acinetobacter baumannii; MRSA: methicillin-resistant Staphylococcus aureus; MICU: medical intensive care unit.

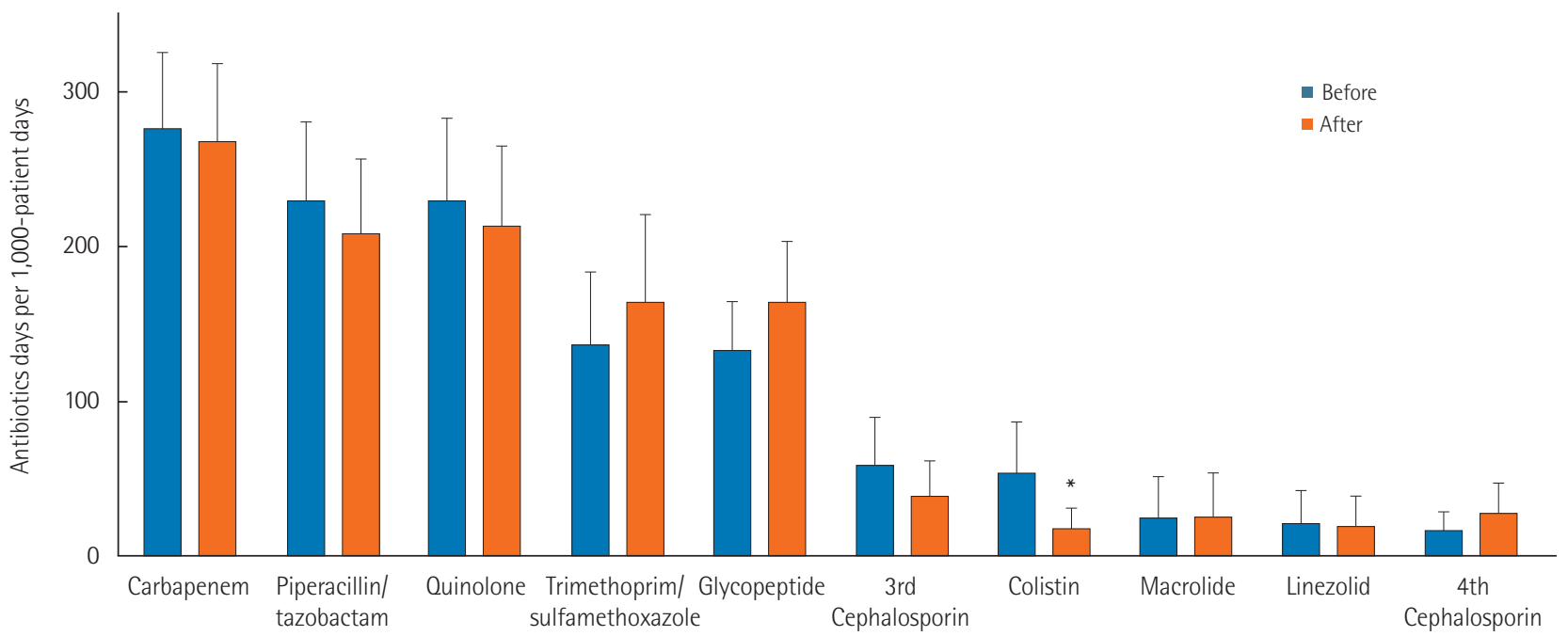

Figure 3. Changes in use of antibiotics before and after the medical intensive care unit relocation. Bars indicate 95\% confidence intervals. *Statistical significance with $\mathrm{P}<0.05$.

\section{Changes in Antibiotic Use}

We reviewed the most commonly used antibiotics and found that carbapenem was most often used, for 275.7 days per 1,000 patient-days before the relocation and 267.8 days after the relocation $(\mathrm{P}=0.827)$. The use of other antibiotics, piperacillin/ tazobactam (229.7 vs. 208.2 days per 1,000 patient-days, $\mathrm{P}=0.547$ ), quinolone (229.2 vs. 213.5 days per 1,000 patient-days, $\mathrm{P}=0.682$ ), glycopeptide (133 vs. 164.1 days per 1,000 patient-days, $\mathrm{P}=0.225$ ), and linezolid (21.6 vs. 19.6 days per 1,000 patient-days, $\mathrm{P}=0.893$ ), did not differ significantly according to the MICU relocation. However, the use of colistin decreased significantly from 53.5 days (95\% CI, 20.3 to 86.7 days) to 18.7 days (95\% CI, 5.6 to 31.7 days) per 1,000 patient-days $(\mathrm{P}=0.027)$ (Figure 3$)$.

\section{Differences in Clinical Outcomes}

We compared the clinical outcomes before and after the MICU relocation. Among the 463 patients included in the study, the overall in-hospital mortality rate was $51.8 \%$. The median length of MICU stay was 5 days (IQR, 2 to 9 days), and the median length of hospital stay was 25 days (IQR, 12 to 46 days). The length of MICU stay (median, 6 vs. 5 days, $\mathrm{P}=0.145$ ) and inhospital mortality ( $52.5 \%$ vs. $47.5 \%, \mathrm{P}=0.382$ ) did not differ significantly between the two groups. However, the length of hospital stay was shortened after the relocation from a median of 29 days (IQR, 14 to 50 days) to a median of 21 days (IQR, 11 to 39 days), which was statistically significant $(P=0.001)$ (Table 2). 
Table 2. Differences in clinical outcomes before and after the MICU relocation

\begin{tabular}{lccc}
\hline Variable & $\begin{array}{c}\text { Before relocation } \\
(n=234)\end{array}$ & $\begin{array}{c}\text { After relocation } \\
(n=229)\end{array}$ & P-value \\
\hline MICU day & $6(3-10)$ & $5(2-9)$ & 0.145 \\
Hospital day ${ }^{a}$ & $29(14-50)$ & $21(11-39)$ & 0.001 \\
In-hospital mortality & $126(52.5)$ & $114(47.5)$ & 0.382
\end{tabular}

Values are presented as median (interquartile range) or number (\%). MICU: medical intensive care unit.

${ }^{a}$ Calculated as calendar days.

\section{DISCUSSION}

In this retrospective, single-center study, relocation of the MICU led to nonsignificant changes in the IR of MDR respiratory microorganisms, including CRAB and MRSA. However, the use of colistin was significantly reduced, and the length of hospital stay was shortened. This is the first study to describe the importance of environmental change to infections by MDR pathogens, including $\mathrm{CRAB}$, one of the respiratory microorganisms most commonly found in ICUs.

The detection rate of MDR respiratory microorganisms and the use of antibiotics in our study are comparable with those reported in previous studies. In a prospective study from Spain [4], the detection rate of MDR respiratory pathogens in ICU patients with pneumonia was $23.9 \%$. In our study, 83 of 463 patients $(17.9 \%)$ had at least one detectable MDR respiratory microorganism, and 34 of 155 patients with pneumonia (21.9\%) had detectable MDR respiratory microorganisms. A prospective study from South Korea reported that the detection rate of CRAB in active surveillance cultures was $15 \%$ [10]. In our study, CRAB was detected in 42 of 463 patients (9.1\%). A prospective study from China reported MRSA incidence to be 13.9 cases per 100 ICU admissions [11], and a retrospective study from Canada reported MRSA incidence to be 13.4-35.6 cases per 1,000 patient-days [12]. Our study revealed 15.8 MRSA cases per 100 admissions (or 22.2 cases per 1,000 patient-days). The use of piperacillin/tazobactam, glycopeptide, and quinolone was similar to that reported in previous studies, but in our center, the use of carbapenem was higher and that of third-generation cephalosporin was lower than previously reported $[11,13]$.

Our results showed nonsignificant changes in the IR of MDR respiratory microorganisms after the relocation. The IR seemed to drop right after the relocation but increased again as time passed, especially in June 2017, when the IR of MDR respiratory microorganisms was the highest in our study period, mostly CRAB and MRSA. Although the exact reason for the sudden outbreak of CRAB and MRSA during June could not be clarified due to the study's retrospective design, some factors could be considered. Among the patients admitted to the MICU during June, a higher proportion were placed on mechanical ventilators (32/43 patients, $74.4 \%$ ) than in other months (282/420 patients, $67.1 \%)$. The duration of ventilator application also tended to be slightly longer in June (mean, 7.3 days) than in the other months (mean, 6.9 days) although that difference was not statistically significant. The application of mechanical ventilators and more ventilator-days has previously been associated with a higher risk of MDR Acinetobacter baumannii colonization [14]. Once colonization has been established, the next patient admitted to the same environment might have been exposed to an elevated risk of developing that infection, especially with Acinetobacter species [15]. Furthermore, contamination of healthcare workers' protective gowns and gloves during routine management of patients can be frequent with Acinetobacter species and MRSA [16]. Those factors could explain why the colonization rate of MDR microorganisms increased with time, even after relocation.

This study demonstrated a significant decrease in colistin use, despite a nonsignificant change in the IR of CRAB from respiratory specimens. This can be explained by the fact that CRAB is a common environmental colonizer $[16,17]$ and that Acinetobacter species are known to cause only $1.5 \%$ to $1.8 \%$ of the nosocomial infections caused by Gram-negative bacilli [18]. It is therefore possible that a significant proportion of patients might have had CRAB "colonization" in their respiratory system rather an infection with "pathogenic" CRAB, resulting in a decrease in the use of colistin.

We also found a shortened hospital stay among patients after the MICU relocation. Because the baseline characteristics (age, sex, APACHE II score, and underlying disease) did not differ significantly before and after the relocation, this difference in clinical outcome could result from changes associated with the MICU relocation, including the reduced use of colistin. Several retrospective studies $[19,20]$ have reported that Acinetobacter baumannii infection is associated with a longer hospital stay. Inappropriate use of antibiotics during the initial days of a hospital-stay is known to be associated with longer hospital stays [21], and that inappropriate use is most commonly associated with Acinetobacter species [22]. Although mortality rates did not differ after the relocation in the present study, there are contradicting reports on differences in mortality rates according to Acinetobacter infections [19,20,23,24]. The inconsistency between the duration of hospitalization and mortality rates could be caused by the low virulence of 
Acinetobacter species [25].

This study has several limitations. First, this was a retrospective study from a single center. Information about recent antibiotics use, adequate antibiotics use, or a history of MDR infections could not be obtained. Second, it was difficult to discriminate between colonizers and pathogens due to the study's retrospective nature. Our results must be replicated in future prospective studies to be generalizable to other clinical settings. Third, due to the center's training curriculum, the annual turnover of resident doctors occurs in March. Therefore, resident doctors in February had nearly 2 years of practice as physicians, whereas those in March had only 1 year of practice and no experience as ICU doctors. That turnover might have affected the clinical outcomes after the MICU relocation [26]. However, the number of MDR pathogens detected in the previous year did not reveal significant monthly variability (Supplementary Table 1), which implies that the annual turnover of residents might not have affected the rates of MDR pathogen detection. Fourth, environmental sampling of the MICU before and after the relocation was not conducted. Collecting that information in future studies could support our results.

In conclusion, the IR of MDR respiratory microorganisms seemed to decrease after the MICU relocation but then increased in subsequent months, thereby leading to a nonsignificant difference in the 4-month IR before and after the relocation. Nonetheless, the use of colistin use was significantly reduced. Although a difference in overall mortality was not observed, length of hospital stay was shortened. Our findings emphasize the importance of environmental contamination control, especially for CRAB.

\section{CONFLICT OF INTEREST}

No potential conflict of interest relevant to this article was reported.

\section{ACKNOWLEDGMENTS}

We would like to thank the staff of the medical intensive care unit and infection control center for their devotion in their daily practice to decrease the incidence rate of multidrug-resistant microorganisms.

\section{ORCID}

Hyung-Jun Kim ～https://orcid.org/0000-0003-1984-8864
EuiSeok Jeong https://orcid.org/0000-0002-8974-1379

Pyoeng Gyun Choe https://orcid.org/0000-0001-6794-7918

Sang-Min Lee https://orcid.org/0000-0002-1388-9318

Jinwoo Lee https://orcid.org/0000-0003-0958-106X

\section{SUPPLEMENTARY MATERIAL}

The online-only Supplement data are available with this article online: https://doi.org/10.4266/acc.2018.00220

\section{REFERENCES}

1. Magill SS, Edwards JR, Beldavs ZG, Dumyati G, Janelle SJ, Kainer MA, et al. Prevalence of antimicrobial use in US acute care hospitals, May-September 2011. JAMA 2014;312:143846.

2. Chung DR, Song JH, Kim SH, Thamlikitkul V, Huang SG, Wang $\mathrm{H}$, et al. High prevalence of multidrug-resistant nonfermenters in hospital-acquired pneumonia in Asia. Am J Respir Crit Care Med 2011;184:1409-17.

3. Corrado RE, Lee D, Lucero DE, Varma JK, Vora NM. Burden of adult community-acquired, health-care-associated, hospital-acquired, and ventilator-associated pneumonia: New York city, 2010 to 2014. Chest 2017;152:930-42.

4. Martin-Loeches I, Torres A, Rinaudo M, Terraneo S, de Rosa F, Ramirez P, et al. Resistance patterns and outcomes in intensive care unit (ICU)-acquired pneumonia: validation of European Centre for Disease Prevention and Control (ECDC) and the Centers for Disease Control and Prevention (CDC) classification of multidrug resistant organisms. J Infect 2015;70:21322.

5. Santos RP, Mayo TW, Siegel JD. Healthcare epidemiology: active surveillance cultures and contact precautions for control of multidrug-resistant organisms: ethical considerations. Clin Infect Dis 2008;47:110-6.

6. Siegel JD, Rhinehart E, Jackson M, Chiarello L; Healthcare Infection Control Practices Advisory Committee. Management of multidrug-resistant organisms in health care settings, 2006. Am J Infect Control 2007;35(10 Suppl 2):S165-93.

7. Urban C, Segal-Maurer S, Rahal JJ. Considerations in control and treatment of nosocomial infections due to multidrug-resistant Acinetobacter baumannii. Clin Infect Dis 2003;36:126874.

8. Aliberti S, Cilloniz C, Chalmers JD, Zanaboni AM, Cosentini $\mathrm{R}$, Tarsia P, et al. Multidrug-resistant pathogens in hospitalised patients coming from the community with pneumonia: a European perspective. Thorax 2013;68:997-9. 
9. Falcone M, Russo A, Giannella M, Cangemi R, Scarpellini MG, Bertazzoni $\mathrm{G}$, et al. Individualizing risk of multidrug-resistant pathogens in community-onset pneumonia. PLoS One 2015; 10:e0119528.

10. An JH, Kim YH, Moon JE, Jeong JH, Kim SH, Kang SJ, et al. Active surveillance for carbapenem-resistant Acinetobacter baumannii in a medical intensive care unit: can it predict and reduce subsequent infections and the use of colistin? Am J Infect Control 2017;45:667-72.

11. Yap FH, Gomersall CD, Fung KS, Ho PL, Ho OM, Lam PK, et al. Increase in methicillin-resistant Staphylococcus aureus acquisition rate and change in pathogen pattern associated with an outbreak of severe acute respiratory syndrome. Clin Infect Dis 2004;39:511-6.

12. Teltsch DY, Hanley J, Loo V, Goldberg P, Gursahaney A, Buckeridge DL. Infection acquisition following intensive care unit room privatization. Arch Intern Med 2011;171:32-8.

13. Thomas Z, Bandali F, Sankaranarayanan J, Reardon T, Olsen KM; Critical Care Pharmacotherapy Trials Network. A multicenter evaluation of prolonged empiric antibiotic therapy in adult ICUs in the United States. Crit Care Med 2015;43:252734.

14. Huang H, Chen B, Liu G, Ran J, Lian X, Huang X, et al. A multicenter study on the risk factors of infection caused by multidrug resistant Acinetobacter baumannii. BMC Infect Dis 2018; 18:11.

15. Nseir S, Blazejewski C, Lubret R, Wallet F, Courcol R, Durocher A. Risk of acquiring multidrug-resistant Gram-negative bacilli from prior room occupants in the intensive care unit. Clin Microbiol Infect 2011;17:1201-8.

16. Morgan DJ, Rogawski E, Thom KA, Johnson JK, Perencevich EN, Shardell M, et al. Transfer of multidrug-resistant bacteria to healthcare workers' gloves and gowns after patient contact increases with environmental contamination. Crit Care Med 2012;40:1045-51.

17. Russotto V, Cortegiani A, Fasciana T, Iozzo P, Raineri SM, Gregoretti $\mathrm{C}$, et al. What healthcare workers should know about environmental bacterial contamination in the intensive care unit. Biomed Res Int 2017;2017:6905450.

18. Gaynes R, Edwards JR; National Nosocomial Infections Surveillance System. Overview of nosocomial infections caused by gram-negative bacilli. Clin Infect Dis 2005;41:848-54.

19. Eberle BM, Schnüriger B, Putty B, Barmparas G, Kobayashi L, Inaba K, et al. The impact of Acinetobacter baumannii infections on outcome in trauma patients: a matched cohort study. Crit Care Med 2010;38:2133-8.

20. Sunenshine RH, Wright MO, Maragakis LL, Harris AD, Song $\mathrm{X}$, Hebden J, et al. Multidrug-resistant Acinetobacter infection mortality rate and length of hospitalization. Emerg Infect Dis 2007;13:97-103.

21. Ibrahim EH, Sherman G, Ward S, Fraser VJ, Kollef MH. The influence of inadequate antimicrobial treatment of bloodstream infections on patient outcomes in the ICU setting. Chest 2000; 118:146-55.

22. Abraham K, Dolman HS, Zimmerman LH, Faris J, Edelman DA, Baylor A, et al. Impact of inappropriate initial antibiotics in critically ill surgical patients with bacteremia. Am J Surg 2016;211:593-8.

23. Wisplinghoff $\mathrm{H}$, Edmond MB, Pfaller MA, Jones RN, Wenzel RP, Seifert H. Nosocomial bloodstream infections caused by Acinetobacter species in United States hospitals: clinical features, molecular epidemiology, and antimicrobial susceptibility. Clin Infect Dis 2000;31:690-7.

24. Abbo A, Carmeli Y, Navon-Venezia S, Siegman-Igra Y, Schwaber MJ. Impact of multi-drug-resistant Acinetobacter baumannii on clinical outcomes. Eur J Clin Microbiol Infect Dis 2007; 26:793-800.

25. Geisinger E, Isberg RR. Interplay between antibiotic resistance and virulence during disease promoted by multidrug-resistant bacteria. J Infect Dis 2017;215(suppl_1):S9-17.

26. Myers L, Mikhael B, Currier P, Berg K, Jena A, Donnino M, et al. The association between physician turnover (the "July Effect") and survival after in-hospital cardiac arrest. Resuscitation 2017;114:133-40. 
Supplementary Table 1. Monthly detection of multidrug-resistant respiratory microorganisms in the medical intensive care unit in the previous year of the study

\begin{tabular}{|c|c|c|c|c|c|c|}
\hline Time & CRAB & CRPA & MRSA & ESBL & SM & Total \\
\hline Nov 2015 & 83 & 2 & 5 & 3 & 4 & 97 \\
\hline Dec 2015 & 80 & 2 & 4 & 2 & 3 & 91 \\
\hline Jan 2016 & 42 & 0 & 11 & 2 & 1 & 56 \\
\hline Feb 2016 & 71 & 5 & 2 & 1 & 3 & 82 \\
\hline Mar 2016 & 58 & 2 & 8 & 0 & 2 & 70 \\
\hline Apr 2016 & 115 & 0 & 7 & 0 & 0 & 122 \\
\hline May 2016 & 82 & 5 & 0 & 3 & 0 & 90 \\
\hline Jun 2016 & 46 & 0 & 9 & 2 & 2 & 59 \\
\hline Jul 2016 & 70 & 0 & 1 & 2 & 2 & 75 \\
\hline
\end{tabular}

Numbers represent cases detected from respiratory specimens.

CRAB: carbapenem-resistant Acinetobacter baumannii; CRPA: carbapenem-resistant Pseudomonas aeruginosa; MRSA: methicillin-resistant Staphylococcus aureus; ESBL: extended-spectrum $\beta$-lactamase; SM: Stenotrophomonas maltophilia. 